\title{
Carbon dioxide clearance using bubble CPAP with superimposed high frequency oscillations in a premature infant lung model with abnormal lung mechanics
}

\author{
Emidio Sivieri ${ }^{1}$, Eric EICHENWALD ${ }^{2}$, David Rub ${ }^{2}$, and Soraya Abbasi ${ }^{2}$ \\ ${ }^{1}$ The Children's Hospital of Philadelphia \\ ${ }^{2}$ Children's Hospital of Philadelphia
}

July 13, 2020

\begin{abstract}
Background: High frequency ( $\mathrm{HF}$ ) oscillatory ventilation has been shown to improve $\mathrm{CO} 2$ clearance in premature infants. In a previous in vitro lung model with normal lung mechanics we demonstrated significantly improved $\mathrm{CO} 2$ washout by $\mathrm{HF}$ oscillation of bubble CPAP (BCPAP). Objective: To examine CO2 clearance in a premature infant lung model with abnormal lung mechanics via measurement of end-tidal CO2 levels (EtCO2) while connected to HF oscillated BCPAP. Design/Methods: A 40mL premature infant lung model with either: normal lung mechanics (NLM): compliance $1.0 \mathrm{~mL} / \mathrm{cmH} 2 \mathrm{O}$, airway resistance $56 \mathrm{cmH} 2 \mathrm{O} /(\mathrm{L} / \mathrm{s})$; or abnormal lung mechanics (ALM): compliance $0.5 \mathrm{~mL} / \mathrm{cmH} 2 \mathrm{O}$, airway resistance $136 \mathrm{cmH} 2 \mathrm{O} /(\mathrm{L} / \mathrm{s})$, was connected to BCPAP with HF oscillation at either 4,6,8,10 or $12 \mathrm{~Hz}$. EtCO2 was measured at BCPAPs of 4,6 and $8 \mathrm{cmH} 2 \mathrm{O}$ and respiratory rates (RR) of 40,60 and 80 breaths/min and $6 \mathrm{~mL}$ tidal volume. Results: HF oscillation decreased EtCO2 levels at all BCPAPs, RRs, and oscillation frequencies for both lung models. Overall mean \pm SD EtCO2 levels decreased ( $p<0.001$ ) from non-oscillated baseline by $19.3 \pm 10.2 \%$ for NLM vs. $14.1 \pm 8.8 \%$ for ALM. CO2 clearance improved for both lung models $(\mathrm{p}<0.001)$ as a function of oscillation frequency and RR with greatest effectiveness at $40-60$ breaths/min and HF at 8-12 Hz. Conclusions: In this in-vitro premature infant lung model, HF oscillation of BCPAP was associated with improved CO2 clearance as compared to non-oscillated BCPAP for both NLM and ALM. The significant improvement in CO2 clearance in an abnormal lung environment is an important step towards clinical testing of this novel respiratory support modality.
\end{abstract}

Carbon dioxide clearance using bubble CPAP with superimposed high frequency oscillations in a premature infant lung model with abnormal lung mechanics.

Emidio M. Sivieri MS.BE ${ }^{1,2}$, Eric C. Eichenwald $\mathrm{MD}^{1,2,3}$, David Rub MD ${ }^{2,3}$, Soraya Abbasi MD ${ }^{1,2,3}$

${ }^{1}$ CHOP Newborn Care at Pennsylvania Hospital, Philadelphia, Pennsylvania

${ }^{2}$ Division of Neonatology, Children's Hospital of Philadelphia, Philadelphia, Pennsylvania

${ }^{3}$ Department of Pediatrics, Perelman School of Medicine, University of Pennsylvania, Philadelphia, Pennsylvania

Keywords: Non-invasive ventilation, $\mathrm{CO}_{2}$ clearance, Lung compliance, Airway resistance, End-tidal carbon dioxide

Short Title : High frequency bubble CPAP: abnormal model lung mechanics

\section{Summary}

Background : High frequency (HF) oscillatory ventilation has been shown to improve $\mathrm{CO}_{2}$ clearance in premature infants. In a previous in vitro lung model with normal lung mechanics we demonstrated significantly 
improved $\mathrm{CO}_{2}$ washout by $\mathrm{HF}$ oscillation of bubble CPAP (BCPAP).

Objective : To examine $\mathrm{CO}_{2}$ clearance in a premature infant lung model with abnormal lung mechanics via measurement of end-tidal $\mathrm{CO}_{2}$ levels $\left(\mathrm{EtCO}_{2}\right)$ while connected to $\mathrm{HF}$ oscillated BCPAP.

Design/Methods : A 40mL premature infant lung model with either: normal lung mechanics (NLM): compliance $1.0 \mathrm{~mL} / \mathrm{cmH}_{2} \mathrm{O}$, airway resistance $56 \mathrm{cmH}_{2} \mathrm{O} /(\mathrm{L} / \mathrm{s})$; or abnormal lung mechanics (ALM): compliance $0.5 \mathrm{~mL} / \mathrm{cmH}_{2} \mathrm{O}$, airway resistance $136 \mathrm{cmH}_{2} \mathrm{O} /(\mathrm{L} / \mathrm{s})$, was connected to BCPAP with HF oscillation at either 4,6,8,10 or $12 \mathrm{~Hz}$. EtCO $\mathrm{t}_{2}$ was measured at BCPAPs of 4,6 and $8 \mathrm{cmH}_{2} \mathrm{O}$ and respiratory rates (RR) of 40,60 and 80 breaths/min and $6 \mathrm{~mL}$ tidal volume.

Results : HF oscillation decreased $\mathrm{EtCO}_{2}$ levels at all BCPAPs, RRs, and oscillation frequencies for both lung models. Overall mean $\pm \mathrm{SD} \mathrm{EtCO}_{2}$ levels decreased $(\mathrm{p}<0.001)$ from non-oscillated baseline by $19.3 \pm 10.2 \%$ for NLM vs. $14.1 \pm 8.8 \%$ for ALM. $\mathrm{CO}_{2}$ clearance improved for both lung models $(\mathrm{p}<0.001)$ as a function of oscillation frequency and RR with greatest effectiveness at 40-60 breaths/min and HF at 8-12 $\mathrm{Hz}$.

Conclusions : In this in-vitro premature infant lung model, HF oscillation of BCPAP was associated with improved $\mathrm{CO}_{2}$ clearance as compared to non-oscillated BCPAP for both NLM and ALM. The significant improvement in $\mathrm{CO}_{2}$ clearance in an abnormal lung environment is an important step towards clinical testing of this novel respiratory support modality.

\section{Introduction}

Noninvasive respiratory support modalities have been increasingly implemented in neonatal intensive care units in an effort to reduce ventilator-induced lung injury. Continuous positive airway pressure (CPAP) and heated and humidified high flow nasal cannula (HFNC) are two commonly used modes of noninvasive support which have recently become the focus of numerous studies evaluating their comparative efficacies. In particular, bubble nasal (BCPAP) has gained renewed interest not only due to its simplicity, but also because the random pressure oscillations caused by the bubbling have been suggested to improve gas exchange through mechanisms of action similar to those observed in high frequency ventilation ${ }^{1,2}$. In this regard, we have recently performed a series of in-vitro tests demonstrating improved carbon dioxide clearance when high frequency oscillations are superimposed onto the supply flow of a HFNC system ${ }^{3,4}$ as well as onto the supply flow of a BCPAP system ${ }^{5}$. The aforementioned studies were exploratory proof-of-concept bench studies using a premature infant lung model having simulated normal, non-diseased, pulmonary mechanics.

In this context, it is known that lung compliance and airway resistance can significantly affect transmission and dynamics of the high frequency pulsations. It is for this reason that further, more detailed, bench measurements are required prior to in-vivo studies or progression to clinical testing.

Therefore, the objective of this current work was to repeat measurements of end-tidal carbon dioxide $\left(\mathrm{EtCO}_{2}\right)$ levels in an infant lung model simulating both normal and moderately affected, or abnormal, lung mechanics while connected to a BCPAP system with superimposed in-line HF oscillations at several oscillation frequencies and over a range of BCPAP settings, and respiratory rates.

\section{Materials and Methods}

Experimental setup and apparatus:

A previously described premature infant lung simulator ${ }^{5}$ consisting of a $40 \mathrm{~mL}$ silicone bellows is illustrated in Figure 1. The lung model was configured with either: normal lung mechanics with compliance of 1.0 $\mathrm{mL} / \mathrm{cmH}_{2} \mathrm{O}$ and airway resistance of $56 \mathrm{cmH}_{2} \mathrm{O} /(\mathrm{L} / \mathrm{s})$; or abnormal lung mechanics with compliance of 0.5 $\mathrm{mL} / \mathrm{cmH}_{2} \mathrm{O}$ and airway resistance of $136 \mathrm{cmH}_{2} \mathrm{O} /(\mathrm{L} / \mathrm{s})$ and connected to a simulated upper airway (3.5 $\mathrm{mm}$ nares diameter). Total instrumented dead space was $4 \mathrm{~mL}$. BCPAP was applied using a Fisher\&Paykel BC163 system with BC3520 infant nasal prongs and supply flow was fixed at $7 \mathrm{~L} / \mathrm{min}$ (Fisher \& Paykel Healthcare Ltd, Auckland, NZ). The $3.5 \mathrm{~mm}$ nasal prongs were positioned to have a snug, leak-free, fit with the $3.5 \mathrm{~mm}$ nares. Oscillation was achieved by interrupting the supply gas by a solenoid pinch valve 
at either $4,6,8,10$ or $12 \mathrm{~Hz}$ with a $40 \%$ on-off duty cycle. To simulate $\mathrm{CO}_{2}$ production, $100 \% \mathrm{CO}_{2}$ was continuously injected at a fixed rate of $12 \mathrm{~mL} /$ minute. $\mathrm{CO}_{2}$ was sampled at the tracheal connection from a continuously circulated side-stream to and from the bellows and through a capnometer (Respironics NM3 monitor equipped with a LoFlo side stream infrared $\mathrm{CO}_{2}$ module, Phillips Corp., Wallingford, CT, USA). Hot-wire anemometers (Bear NVM-1, Bear Medical Systems, Riverside CA, USA) were used to measure the lung model bi-directional flow at the simulated tracheal level and also the oscillatory flow entering the nasal cannula. Airway pressure at the simulated tracheal level and applied BCPAP pressure proximal to the nasal prongs were measured using Biopac TSD-160D pressure transducers (range $\pm 75 \mathrm{cmH}_{2} \mathrm{O}$, combined linearity and hysteresis $\pm 0.05 \%, 100 \mathrm{~Hz}$ dynamic response, Biopac Systems, Goleta, CA).

The capnometer was calibrated with a calibration gas mixture consisting of $4.9 \pm 0.1 \% \mathrm{CO}_{2}, 14.9 \% \mathrm{O}_{2}$ and balance $\mathrm{N}_{2}$ (Gasco Affiliates, Oldsmar Fla., USA). Pressure was calibrated using a digital manometer $( \pm 0.27 \%$ NIST accuracy, Extech Instruments, Nashua, N.H., USA). Hot-wire anemometers were calibrated using a precision rotameter (range $\pm 9 \mathrm{~L} / \mathrm{min}, \pm 1 \%$ accuracy, Aalborg Instruments, Orangeburg, NY, USA). Frequency response of the flow sensors was tested in our laboratory using a calibrated square wave flow signal and were found to maintain accuracy with a flat frequency response to at least $18 \mathrm{~Hz}$. All analog signals were digitally converted at $200 \mathrm{~Hz}$ and 16-bit resolution and recorded with a data acquisition system (Biopac MP150 and Acqknowledge v4.2 software).

\section{Experimental Protocol:}

The breathing simulator with normal compliance and resistance was set to simulated spontaneous breathing at a fixed sinusoidal tidal volume of $6.0 \mathrm{~mL}$ and inspiratory-to-total breath time ratio $\left(\mathrm{T}_{\mathrm{i}} / \mathrm{T}_{\mathrm{t}}\right)$ of 0.4 for all measurements. An initial respiratory rate (RR) was set at 40 breaths/minute and the BCPAP level was set to $4 \mathrm{cmH}_{2} \mathrm{O}$ with no applied high frequency oscillation. After the $\mathrm{EtCO}_{2}$ signal reached a stable baseline level, the solenoid valve was activated to operate at $4 \mathrm{~Hz}$ and the $\mathrm{EtCO}_{2}$ was again allowed to equilibrate, after which, oscillation was stopped and $\mathrm{EtCO}_{2}$ was allowed to equilibrate back to the baseline value. This sequence was repeated at CPAP settings of 4,6 and $8 \mathrm{cmH}_{2} \mathrm{O}$ and at each of the oscillation frequencies of 4,6,8, 10 and $12 \mathrm{~Hz}$ and again using RRs of 60 and 80 breaths/minute. The entire sequence was then performed using the lung model configured with the abnormal compliance and resistance. All measurements were performed in triplicate.

Data analysis:

Student's paired-T tests and analysis of variance tests were used to test for differences in oscillatory and non-oscillatory $\mathrm{EtCO}_{2}$ values across the three levels of CPAP settings, five oscillation frequencies and three RRs for the normal and abnormal lung model configurations. A p-value of $<0.05$ was considered indicative of statistical significance. $\mathrm{EtCO}_{2}$ values are reported as mean \pm SD. SPSS version 19 (IBM SPSS Statistics, IBM Corp. Armonk, NY, USA) was used for statistical analysis.

\section{Results}

A representative recording of tidal $\% \mathrm{CO}_{2}$, airway flow and tidal volume signals during the transition from non-oscillation, i.e. baseline BCPAP only, to $8 \mathrm{~Hz}$ oscillation mode is shown in Figure 2 and illustrates equilibration of the $\mathrm{EtCO}_{2}$ to a lower level after oscillation is applied. Random oscillations due only to the bubbling may be observed on the airflow signal in the baseline expanded time-scale portion of the figure. These small oscillations were determined to have a frequency content between 6 and $18 \mathrm{~Hz}$.

High frequency oscillation resulted in decreased $\mathrm{EtCO}_{2}$ levels at all CPAP settings, RRs, and applied frequencies for both the normal and abnormal lung models as summarized in Figure 3. The overall mean \pm SD $\mathrm{EtCO}_{2}$ levels decreased significantly from non-oscillated baseline by $19.3 \pm 10.2 \%(\mathrm{p}<0.001)$ for the normal lung mechanics model vs. a lesser change of $14.1 \pm 8.8 \%(\mathrm{p}<0.001)$ for the abnormal model. This difference in $\mathrm{CO}_{2}$ clearance between normal vs. abnormal lung mechanics was found to be statistically significant (p $=0.011$.

The set BCPAP level had no significant effect on the observed $\mathrm{EtCO}_{2}$ responses, whereas $\mathrm{CO}_{2}$ clearance 
improved significantly for both lung models as a function of oscillation frequency $(\mathrm{p}<0.001)$ and RR ( $\mathrm{p}$ $<0.001$ ), as detailed in Figure 4. Oscillation frequencies between 8 and $12 \mathrm{~Hz}$ demonstrated maximum effectiveness when the lung models were operated at 40 and 60 breaths $/$ min. The greatest $\mathrm{EtCO}_{2}$ reductions, averaged over all three BCPAP levels, occurred using a RR of 40 breaths/min as shown in Figure $4 \mathrm{~A}$ and 4B with downward peaks at $-36.8 \%$ using $8 \mathrm{~Hz}$ oscillation for the normal lung model and at $-30.1 \%$ using 12 $\mathrm{Hz}$ oscillation for the abnormal lung model.

Using measured values of the small amplitude volume oscillations (Vt) resulting from high frequency interruption of the BCPAP supply flow (as shown in the expanded portion of the tidal volume signal in Figure 2) we plotted the theoretical gas transport relationship for high frequency oscillation ${ }^{6,7}$ as given by: $\mathrm{DCO}_{2}$ [?] $\mathrm{Vt}^{2} \times \mathrm{F}$, for each applied frequency $(\mathrm{F})$ and for each BCPAP level as shown in Figure 5. Peak $\mathrm{DCO}_{2}$ values occurred at 8 and $12 \mathrm{~Hz}$ for the normal lung mechanics model and at $8 \mathrm{~Hz}$ for the abnormal model.

\section{Discussion}

Among the several noninvasive respiratory support modalities for preterm infants, nasal CPAP continues to be a first choice among clinicians in lieu of intubation. Nonetheless, CPAP failure rates have persisted to be near $50 \%^{8,9}$.

This study was undertaken as a continuation of previous work performed in this laboratory in which a novel in-line flow interrupter device was evaluated to determine its efficacy as a simple means of improving carbon dioxide elimination in a nasal CPAP system ${ }^{3,5}$. We previously demonstrated that superimposing high frequency oscillation had better performance in improving carbon dioxide clearance when applied to a BCPAP system compared to the commonly used variable-flow type of nasal CPAP.

In the present study we repeated these measurements for a lung model with abnormal lung mechanics having a relatively stiffer compliance $\left(0.5 \mathrm{~mL} / \mathrm{cmH}_{2} \mathrm{O}\right)$ and a higher airway resistance $\left(136 \mathrm{cmH}_{2} \mathrm{O} /(\mathrm{L} / \mathrm{s})\right)$; these values being more consistent with those of an infant to be placed on CPAP support. Applying high frequency to this abnormal lung model we demonstrated a significant overall $\mathrm{EtCO}_{2}$ reduction of $14.1 \%$ from non-oscillated baseline levels; whereas the normal lung mechanics model resulted in an overall $\mathrm{EtCO}_{2}$ reduction of $19.3 \%$ from baseline. This relatively small but significant difference is most likely due to smaller amplitude HF oscillations in the stiffer and more restricted lung as predicted by the lower $\mathrm{DCO}_{2}$ curves shown in Figure 5B when compared to values for the normal lung model shown in Figure 5A.

As in our previous studies, we postulate that the improvement in $\mathrm{CO}_{2}$ clearance in both lung models is due to a synergistic combination of the random BCPAP bubble oscillations with the high frequency oscillations superimposed onto the BCPAP supply flow. This concept is reinforced by a power spectral density plot of the random bubble oscillations (Figure 6) showing a bubble frequency spectrum with dominant power peaks which closely coincide with the optimum applied frequencies where $\mathrm{EtCO}_{2}$ reduction is greatest, specifically at 8 and $12 \mathrm{~Hz}$ (Figure 4).

In a bench study by Mukerji et al, a significant reduction in $\mathrm{CO}_{2}$ clearance rate was also demonstrated for a low compliance compared to a 4-times higher compliance term newborn lung model in which high frequency nasal mechanical ventilation was compared to positive pressure ventilation. They did not observe any $\mathrm{CO}_{2}$ elimination under non-oscillated nasal CPAP, the non-ventilatory mode of the study's commercially available neonatal mechanical ventilator ${ }^{10}$.

Our results are consistent with well-known principles of high frequency, small amplitude, oscillation in a tubular airstream enabling net longitudinal gas transport through several facilitated diffusion mechanisms 11,12. This effect is especially relevant for premature infants with relatively high dead space to tidal volume ratio compared to larger, more mature infants ${ }^{13,14}$, enabling gas exchange with tidal volumes smaller than the anatomic dead space.

In this in vitro study we have demonstrated that the addition of oscillations at a frequency near the dominant bubbling frequency may result in a synergistic effect to further enhance gas exchange. It is proposed that the frequency and duty-cycle of the applied oscillations may be "tuned" for a particular bubble CPAP system to 
optimize gas exchange in infants according to their degree of abnormal lung mechanics. Furthermore, that the main active components of this prototype device (waveform generator, amplifier and solenoid valve) may be integrated into a single, stand-alone device with adjustable frequency, duty-cycle and amplitude which could be adapted for use with any nasal CPAP system and in place of dedicated non-invasive high frequency oscillatory ventilators.

The strengths of this study are the highly repeatable measurements under easily controlled model lung dynamics, namely, compliance, airway resistance, $\mathrm{FRC}$ and $\mathrm{CO}_{2}$ production rate which are all experimental conditions not easily obtainable or feasible in in-vivo measurements. Nonetheless, study limitations include the fact that this is an in vitro bench study using a simplified lung model that cannot account for multifaceted in-vivo respiratory physiology nor the complex geometry of neonatal nasal and upper airways and airflow dynamics. For example, soft-tissue structures in the human respiratory pathway may dampen the high frequency oscillations such as to limit any enhanced carbon dioxide clearance effect ${ }^{15}$. Furthermore, measurements in this study were made under leak-free cannula and closed-mouth conditions, whereas we demonstrated in a previous carbon dioxide washout study significantly decreased washout times for both open- and partially-open mouth conditions ${ }^{16}$. Notably, both prong size and insertion depth have also been shown to affect $\mathrm{CO}_{2}$ washout times and airway pressures ${ }^{17,18}$.

Conclusion

In this exploratory bench study, we have demonstrated improved carbon dioxide clearance when high frequency oscillation is applied to a bubble CPAP system tested on both an infant lung model having close to normal lung mechanics and an abnormal lung model with pulmonary characteristics closer to those of a premature infant requiring respiratory support. The ability to improve $\mathrm{CO}_{2}$ clearance in an abnormal lung environment, may prove this novel device to be effective in reducing CPAP failure. Nonetheless, given the complexity of the neonatal respiratory system, it is clear that clinical studies are needed to fully evaluate this modification of nasal CPAP support systems.

Finally, we speculate that the low cost and low complexity of this simplified device may be useful in lowand middle-income settings with limited access to newer respiratory support equipment and where the use of basic, tube-in-bottle bubble CPAP has been shown to be very effective in improving survival, particularly in very low birthweight infants ${ }^{19-21}$.

\section{Acknowledgments}

The authors gratefully acknowledge Fisher \& Paykel Healthcare for providing bubble CPAP equipment. We have not received salary or other personal benefits from, nor hold stock in, the company. The study was designed, conducted, data analyzed and manuscript prepared independently and with no input from Fisher \& Paykel. The authors also gratefully acknowledge Toni Mancini RN, BSN, CCRC for critical review of the manuscript.

\section{References}

1. Lee KS, Dunn MS, Fenwick M, Shennan AT. A comparison of underwater bubble continuous positive airway pressure with ventilator-derived continuous positive airway pressure in premature neonates ready for extubation. Biol Neonate 1998;73:69-75.

2. Pillow JJ, Hillman N, Moss TJ, Polglase G, Bold G, Beaumont C, Ikegami M, Jobe AH. Bubble continuous positive airway pressure enhances lung volume and gas exchange in preterm lambs. Am J Respir Crit Care Med 2007;176(1):63-69.

3. Sivieri EM, Eichenwald E, Bakri SM, Abbasi S. Effect of high frequency oscillatory high flow nasal cannula (HFNC) on carbon dioxide clearance in a premature infant lung model: A bench study. Pediatr Pulmonol 2019;54(4):436-443.

4. Rub DM, Sivieri EM, Abbasi S, Eichenwald E. Effect of high-frequency oscillation on pressure delivered by high flow nasal cannula in a premature infant lung model. Pediatr Pulmonol 2019; 54(11):1860-1865.

5. Sivieri EM, Eichenwald EC, Rub DM, Abbasi S. An in-line high frequency flow interrupter applied to 
nasal CPAP: improved carbon dioxide clearance in a premature infant lung model. Pediatr Pulmonol 2019;54(12):1974-1981.

6. Pillow JJ, Wilkinson MH, Neil HL, Ramsden CA. In vitro performance characteristics of high-frequency oscillatory ventilators. Am J Respir Crit Care Med 2001;164(6):1019-1024.

7. Keszler M, Durand DJ. Neonatal high-frequency ventilation: past, present, and future. Clin Perinatol 2001;28(3):579-607.

8. Dunn MS, Kaempf J, de Klerk A, de Klerk R, Reilly M, Howard D, Ferrelli K, O'Conor J, Soll RF, Vermont Oxford Network DRMSG. Randomized trial comparing 3 approaches to the initial respiratory management of preterm neonates. Pediatrics 2011;128:e1069-e1076.

9. Morley CJ, Davis PG, Doyle LW, Brion LP, Hascoet JM, Carlin JB, COIN Trial Investigators. Nasal CPAP or intubation at birth for very preterm infants. N Engl J Med 2008;358(7):700-708.

10. Mukerji A, Finelli M, Belik J. Nasal high-frequency oscillation for lung carbon dioxide clearance in the newborn. Neonatology 2013;103(3):161-165.

11. Chang HK. Mechanisms of gas transport during ventilation by high-frequency oscillation. J Appl Physiol 1984;56:553-563.

12. Pillow JJ. High-Frequency oscillatory ventilation: mechanisms of gas exchange and lung mechanics. Crit Care Med 2005;33(3 Suppl):S135-S141.

13. Neumann RP, Pillow JJ, Thamrin C, Larcombe AN, Hall GL, Schulzke SM. Influence of gestational age on dead space and alveolar ventilation in preterm infants ventilated with volume guarantee. Neonatology 2015;107(1):43-49.

14. Wenzel U, Wauer RR, Schmalisch G. Comparison of different methods for dead space measurements in ventilated newborns using CO2-volume plot. Intensive Care Med 1999;25(7):705-713.

15. Yoder BA, Albertine KH, Null DM. High-frequency ventilation for non-invasive respiratory support of neonates. Seminars in Fetal and Neonatal Medicine 2016;21(3):162-173.

16. Sivieri E, Foglia E, Abbasi S. Carbon dioxide washout during high flow nasal cannula versus nasal CPAP support: An in-vitro study. Pediatr Pulmonol 2017;52:792-798.

17. De Luca D, Piastra M, Pietrini D, Conti G. Effect of amplitude and inspiratory time in a bench model of non-invasive HFOV through nasal prongs. Pediatr Pulmonol 2012;47:1012-1018.

18. Sivieri EM, Gerdes JS, Abbasi S. Effect of HFNC flow rate, cannula size, and nares diameter on generated airway pressures: an in vitro study. Pediatr Pulmonol 2013;48(5):506-514.

19. Martin S, Duke T, Davis P. Efficacy and safety of bubble CPAP in neonatal care in low and middle income countries: a systematic review. Arch Dis Child Fetal Neonatal Ed 2014;99(6):F495-F504.

20. Kawaza K, Machen HE, Brown J, Mwanza Z, Iniguez S, Gest A, O'Brian Smith E, Oden M, RichardsKortum RR, Molyneux E. Efficacy of a low-cost bubble CPAP system in treatment of respiratory distress in a neonatal ward in Malawi. Malawi Med J 2016;28(3):131-137.

21. Won A, Suarez-Rebling D, Baker AL, Burke TF, Nelson BD. Bubble CPAP devices for infants and children in resource-limited settings: review of the literature. Paediatr Int Child Health 2019;39(3):168176.

\section{Hosted file}

Figure legends HF Bubble CPAP.docx available at https://authorea.com/users/341926/articles/ 468804-carbon-dioxide-clearance-using-bubble-cpap-with-superimposed-high-frequencyoscillations-in-a-premature-infant-lung-model-with-abnormal-lung-mechanics 

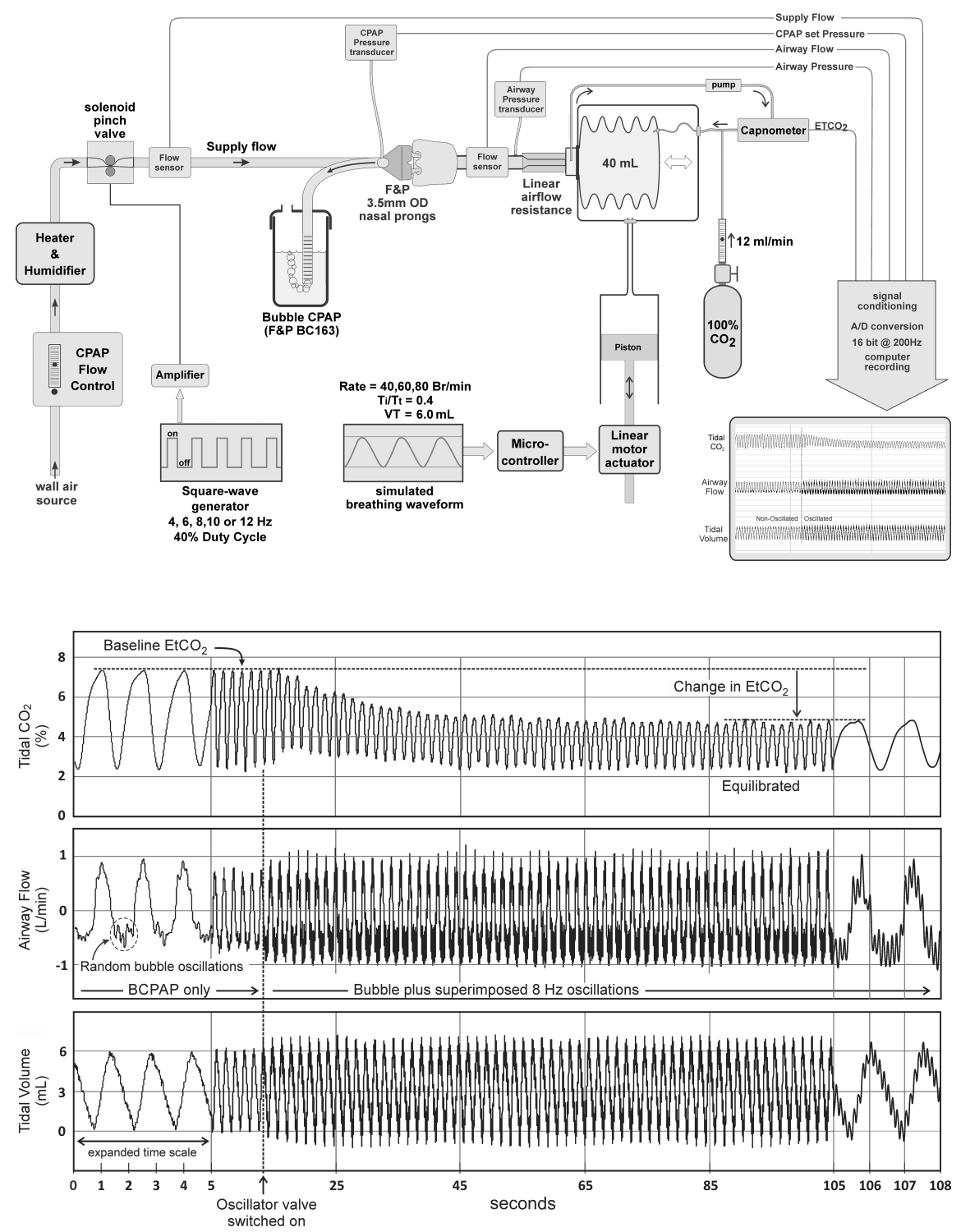

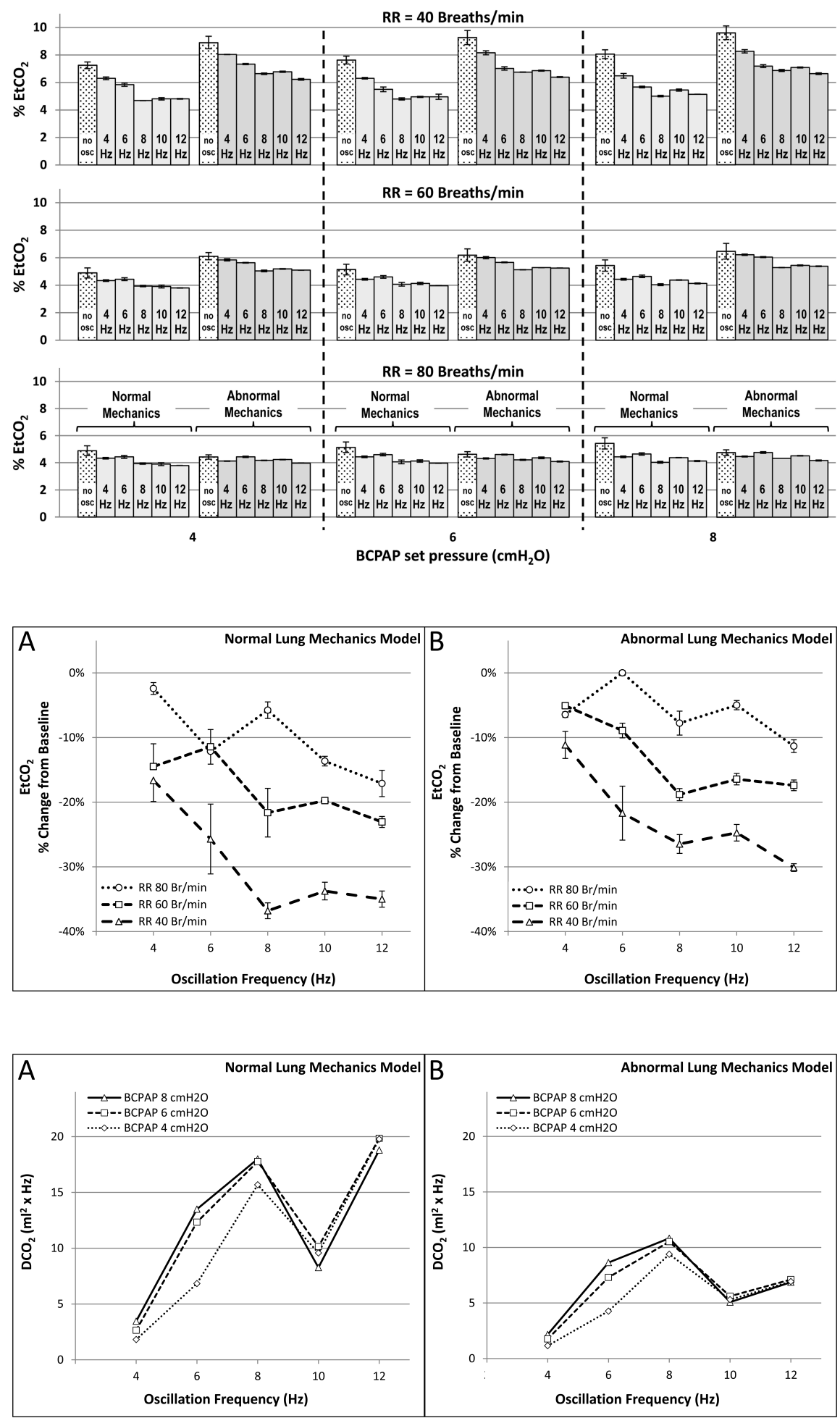


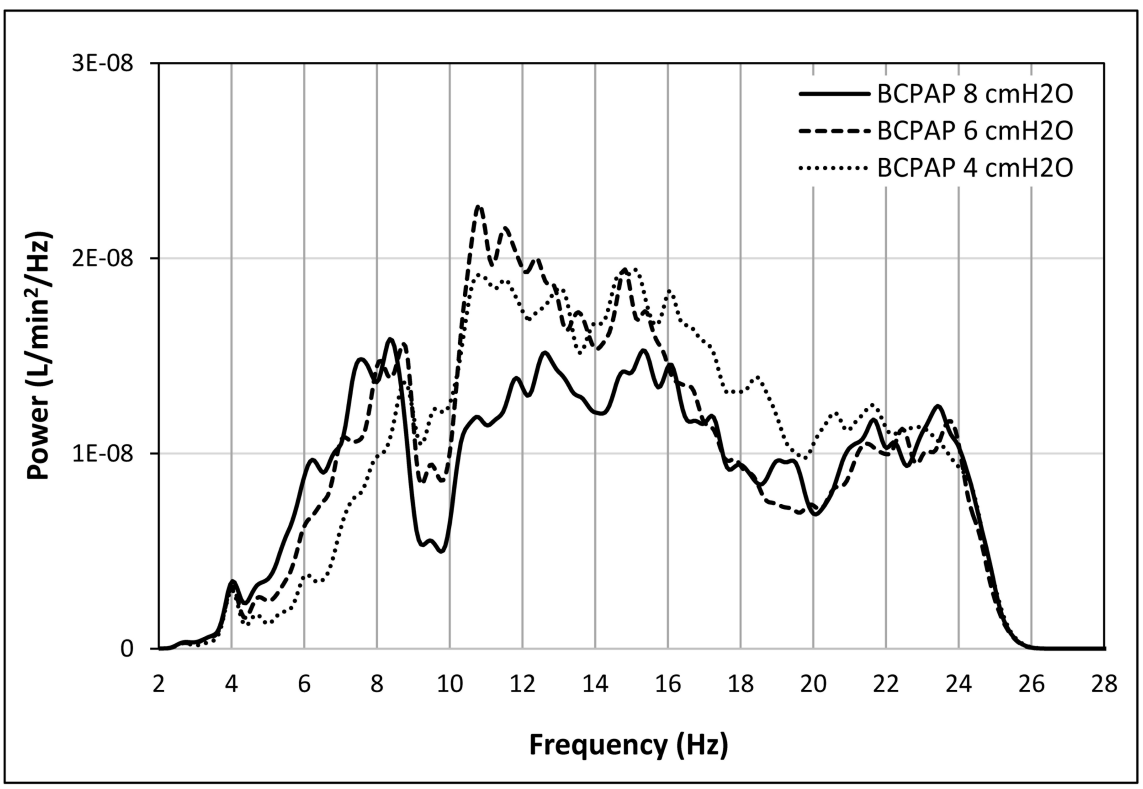

